\title{
Effects of the combined extracts of Herba Epimedii and Fructus Ligustrilucidi on airway remodeling in the asthmatic rats with the treatment of budesonide
}

\author{
Xiufeng Tang, Honglei Nian, Xiaoxi Li, Yan Yang, Xiujuan Wang, Liping Xu, Haotian Shi, Xinwei Yang
} and Renhui Liu* (D)

\begin{abstract}
Background: Asthma is characterized by chronic airway inflammation, leading to structura1 changes in the airway, collectively termed airway remodeling. Airway remodeling is thought to contribute to airway hyper responsiveness and irreversible airflow limitation. The combination of Herba Epimedii (HE) and Fructus Ligustri Lucidi (FLL) decoction and the systemic administration of glucocorticoids (GC) had a synergistic inhibitory action on airway inflammation in the asthmatic model rats. However, the effects of the combination on airway remodeling have not been studied and compared. In the present study, we investigated the effects of the co-administration of combined extracts of HE and FLL with inhaled GC (budesonide) on airway remodeling in the rat asthmatic model induced by ovalbumin (OVA).

Methods: Male Sprague-Dawley rats were sensitized to intraperitoneal OVA followed by repetitive OVA challenge for 7 weeks. Treatments included extracts of HE and FLL (Extracts for short, $100 \mathrm{mg} / \mathrm{kg}$ by gastric perfusion), budesonide (1 mg budesonide suspension in $50 \mathrm{ml}$ sterile physiological saline, 3 rats in an ultrasonic nebulizer by nebulized inhabation with a flow of $1.6 \mathrm{ml} / \mathrm{min}$ for $30 \mathrm{~min}$ ), and co-administration of extracts of HE and FLL with budesonide (Co-administration for short) for 4 weeks. Lung histomorphometry and bronchoalveolar lavage fluid (BALF) cell count were assessed $24 \mathrm{~h}$ after the final OVA challenge. Levels of interleukin (IL)-4, IL-5 and IgE were measured by ELISA. Expressions of Collagen I and Collagen III were tested by immunohistology. Expressions of transforming growth factor (TGF) - $\beta 1$, TGF- $\beta 2$ and Smads mRNA were measured by quantitative real-time PCR.
\end{abstract}

Results: Extracts, budesonide and Co-administration significantly reduced allergen-induced increases in the serum levels of IL-4, IL-5 and IgE, the number of eosinophils in BALF, goblet cell hyperplasia, Collagen III integral optical density (IOD) and the mRNA expression of TGF- $\beta 2$ and Smad2. Extracts and Co-administration could depress the IOD level of Collagen I and the positive area of Collagen I and Collagen III. Budesonide and Co-administration significantly alleviated the thickening of airway wall. Only Co-administration significantly decreased collagen deposition according to the morphometry of Masson's-stained lung sections, the thickening of airway smooth muscle layer, the number of lymphocytes in BALF and the mRNA expression of TGF- $\beta 1$ and Smad3, and this was associated with a significant increase in levels of Smad7 mRNA.

Conclusions: The findings suggested that the combination of budesonide and the herbal extracts had a better synergistic effect on airway remodeling in OVA-reduced asthma rats than the single use of budesonide.

Keywords: Herba Epimedii, Fructus Ligustri Lucidi, Airway remodeling, Asthma model

\footnotetext{
* Correspondence: gzblrh45@ccmu.edu.cn

Beijing Key Lab of TCM Collateral Disease Theory Research, School of

Traditional Chinese Medicine, Capital Medical University, No.10 Xitoutiao,

Youanmenwai, Fengtai District, Beijing 100069, China
}

(c) The Author(s). 2017 Open Access This article is distributed under the terms of the Creative Commons Attribution 4.0 International License (http://creativecommons.org/licenses/by/4.0/), which permits unrestricted use, distribution, and reproduction in any medium, provided you give appropriate credit to the original author(s) and the source, provide a link to the Creative Commons license, and indicate if changes were made. The Creative Commons Public Domain Dedication waiver (http://creativecommons.org/publicdomain/zero/1.0/) applies to the data made available in this article, unless otherwise stated. 


\section{Background}

Bronchial asthma has become one of the most common health problems in the world, especially within industrialized societies [1, 2]. Increasing evidences suggest that asthma is a chronic inflammatory disorder of the airways in which many cells and cellular elements play important roles and is characterized by the infiltration of eosinophils and dominant in helper T cells (Th) 2 cytokines [3-5]. The frequent occurrence of injury and repair initiated by chronic inflammation could lead to structura1changes in the airway, collectively termed airway remodeling. Airway remodeling is characterized by airway wall thickening, subepithelial fibrosis, increased smooth muscle mass, angiogenesis and increased mucous glands [6, 7]. Generally, airway remodeling is thought to contribute to airway hyper-responsiveness and irreversible airflow limitation.

All recent consensus statements on asthma advocate aggressive treatment of airway inflammation [8, 9]. Glucocorticoids (GCs), especially inhaled GCs, remain the cornerstone of asthma management because of the most effective anti-inflammatory effect, tolerability and rapid onset of action [10]. Prolonged low dose administration of inhaled GC is generally considered safe, although there are considerable concerns about the longterm effects of GC among some consumers, particularly children and the GCs-resistant asthmatic patients [11]. Severe asthma has a distinct pathophysiology including airway remodeling that contributes to the decreased effectiveness of standard therapy. However, when moderate or high doses are required to control symptoms, adverse effects such as growth stunting in children [12], suppression of the hypothalamic-pituitary-adrenal (HPA) axis [13, 14], and osteopenia [15] may be observed. For these reasons, effective therapies that are targeted at severe asthma and that can inhibit asthma airway remodeling are needed.

Traditional Chinese medicine (TCM) has a unique advantage in relieving and curing asthma $[16,17]$. There are a number of anti-asthma herbal formulas recorded in TCM literatures and used in practice, but evidencebased researches into their efficacy and mechanisms of efficacy are still in their infancy. According to the theory of TCM, kidney-yang deficiency syndrome is one of the most common syndromes seen in asthmatics and may run through the entire process of asthma [18-20]. TCM doctors often follow the principle of strengthening kidney-yang to treat asthma. Because the syndrome of the asthmatic patient with long-term or high-dose administration of GC is similar to the clinical signs of the kidneyyang deficiency syndrome [21, 22], the herbal medicines of replenishing kidney were often use to prevent the adverse effects of GC when GC was prescribed to treat asthma for a long term or at a high dose by TCM doctor.
Professor Huiguang $X u$, a famous doctor of TCM, has used the decoction of Herba Epimedii (HE) and Fructus Ligustri Lucidi (FLL) to treat kidney-yang deficiency syndrome of asthma, or to combine with GC for the treatment of asthma for almost 50 years [23, 24]. HE and FLL, documented in Chinese ancient medicinal literatures, have strong actions of replenishing kidney-yang and kidney-yin, respectively $[25,26]$. In our previous researches, we established an asthma model in rats by repetitive ovalbumin (OVA)-challenge which replicated many of the features of the human disease asthma with a high degree of fidelity, and demonstrated that the decoction of HE and FLL in combination with administration of systemic GC (dexamethasone) had a better anti-inflammatory effect on the asthmatic model rats, and could prevent the inhibition of HPA axis and loss of bone mass, compared with the administration of dexamethasone [27-29]. However, we found that the effect of dexamethasone on the mRNA expression of glucocorticoids receptor (GR) isoforms in lung tissue was not consistent with the protein content of GR isoforms, suggesting that inhaled GC was less likely to produce GC resistance than systemic administration of GC. So we choose budesonide as a representative drug for inhaled $\mathrm{GC}$ to research the effects of EL on the asthmatic rats with budesonide intervention. This study aimed to compare the effects of the extracts of HE and FLL, inhaled $\mathrm{GC}$, and the combination of the herbal extracts with inhaled GC on airway inflammation and remodeling in asthmatic rat, and provide basis for the combination of Chinese and Western medicine in the treatment of asthma.

\section{Methods}

\section{Preparation of herbal extracts}

$\mathrm{HE}$, the dried leaf of Epimediium brevicornu Maxin. and FLL, the dried mature seed of Ligustrum lucidum Ait., were purchased from Beijing Tongrentang pharmaceutical Co. Ltd., China, and authenticated by an expert herbalist at Capital Medical University. They were stored in a dry and sealed container at $4{ }^{\circ} \mathrm{C}$. Preparation of HE and FLL extracts were performed according to the methods described before [30].

\section{Animals}

Fifty male Sprague-Dawley rats, weighing 120 to130 g with the average age of four or five weeks, were purchased from Vital River Laboratory Animal Technology Co. Ltd. (Beijing, China). The experiment complied with the Animal Management Rule of the Ministry of Public Health, China, and the experimental protocol was approved by the Animal Care Committee of Capital Medical University, Beijing, China. All the animals were cared for in the Experimental Animal Center of Capital 
Medical University. During the whole experiment, the animals were housed in stainless cages (three rats per cage) at conventional controlled conditions (temperature of $23 \pm 2{ }^{\circ} \mathrm{C}$, relative humidity of $50 \pm 10 \%, 12$-h lightdark cycle). They were allowed for free access to the standard laboratory food and tap water.

\section{Experimental protocol}

After acclimatization for 7 days, the rats were randomly assigned into the following five groups ( $n=10$ per group): normal control group (Control), asthma model group (Asthma), budesonide group (Budesonide), group of herbal extracts (Extracts), and group of co-administration of budesonide with herbal extracts (Co-administration).

OVA sensitization and challenge protocols were performed according to the methods of Yang et al. [31] with certain modifications as described below. All the rats with the exception of those in the control group were actively sensitized with an intraperitoneal (ip) injection and subcutaneous injection of $1 \mathrm{mg}$ OVA (Grade II; Sigma, USA) and $100 \mu \mathrm{g}$ aluminum hydroxide (Sigma) in $1 \mathrm{ml}$ sterile physiological saline. After seven days, the same procedure was repeated. After another seven days, the OVA-sensitized rats were exposed to $1 \%$ aerosolized OVA (1 g OVA in $100 \mathrm{ml}$ sterile physiological saline in an ultrasonic nebulizer) for $30 \mathrm{~min}$ once a day. Three weeks later, the OVA-sensitized rats were challenged twice a week and treated every day. Rats in the budesonide and co-administration groups were exposed to budesonide suspension for inhalation (AstraZeneca Pty Ltd., AUS; $1 \mathrm{mg}$ budesonide suspension in $50 \mathrm{ml}$ sterile physiological saline, three rats in an ultrasonic nebulizer with a flow of $1.6 \mathrm{ml} / \mathrm{min}$ ) for $30 \mathrm{~min}$. Rats in the Extracts and Co-administration groups were administered with the combined extracts of HE and FILL via oral gavage at the dose of $100 \mathrm{mg} / \mathrm{kg}$ body weight. The rats in the normal control group and asthma model group were given with the same volume of distilled water. On the 64th day, all the rats were anesthetized with $25 \%$ ethyl carbamate $(4 \mathrm{~mL} / \mathrm{kg}, \mathrm{ip})$.

\section{Cell count of BALF cytospin}

The right lungs were lavaged 3 times with $3 \mathrm{~mL}, 3 \mathrm{~mL}$ and $4 \mathrm{~mL}$ ice-cold saline using a tracheal cannula and a $5 \mathrm{~mL}$ polyethylene syringe. The cell-debris pellets of bronchoalveolar lavage fluid (BALF) samples were collected after centrifugation (500 rpm, $5 \mathrm{~min}$, and $4{ }^{\circ} \mathrm{C}$ ). A differential cell count was performed on cytospin by Wright-Giemsa staining with the Nikon ECLIPSE 80i biomicroscope and NIS-Elements BR 3.2 image analysis system (Nikon, Japanese). The number of lymphocytes and eosinophils in 200 cells was counted.

\section{Lung histopathology and morphometry}

The middle lobe of the left lungs was removed and fixed in $4 \%$ neutral-buffered paraformaldehyde solution in PBS (pH 7.4) for $24 \mathrm{~h}$, embedded in paraffin and then routinely processed. Lung tissue sections $(4 \mu \mathrm{m})$ were stained with hematoxylin and eosin (H\&E), Masson's trichrome and periodic-acid schiff (PAS) stain. A minimum of 3 bronchi (luminal diameter, 150-500 $\mu \mathrm{m}$ ) were analyzed per rat for various parameters using an imageanalyzing computer system (NIS-Elements BR 3.2; Nikon, Japan).

H\&E-stained lung sections were used mainly for the assessment of peribronchial inflammation and airway remodeling. According to the method of Ming Chen et al. [32], 3 bronchioles with 150-200 $\mu \mathrm{m}$ inner diameter were selected and counted in each slide. The perimeter of basement membrane ( $\mathrm{Pbm})$, total area of airway wall (Wat) and area of smooth muscle (Wam) were measured and the average was calculated. Pbm was used for normalization of Wat and Wam. Then we used the ratios of Wat to $\mathrm{Pbm}$ (Wat/Pbm) and Wam to $\mathrm{Pbm}$ (Wam/Pbm) to evaluate airway remodeling.

Masson's trichrome-stained sections were used for identification of subepithelial collagen [33, 34]. Briefly, the epithelial basement membranes (diameter $\geq 250 \mu \mathrm{m}$ ) were selected, and the basement membrane perimeter and collagen fiber area (stained in blue) beneath the basement membrane in $20 \mu \mathrm{m}$ depth were measured. The mean score of the fibrotic area divided by the basement membrane perimeter in every rat was calculated.

PAS-stained sections were used for observing the goblet cells $[35,36]$. The length of the epithelial basement membrane of the bronchus of $\geq 500 \mu \mathrm{m}$ was selected, and the number of PAS-positive goblet cells was counted standardized to $\mathrm{Pbm}$ to account for variations in bronchiole diameters. The area of goblet cells within the epithelial lining area of $100 \mu^{2}$ was analyzed for each airway.

\section{Enzyme linked immunosorbent assy (ELISA)}

Blood samples were collected and separated simultaneously using a centrifuge (Biofuge 15R, Heraeus Sepatech, USA), and serum was collected finally and stored at $-80^{\circ}$ $\mathrm{C}$ prior to assay. The serum levels of IL-4, IL-5 and IgE were measured using rat ELISA kits (Cusabio Biotech CO., Ltd., Wuhan, China) by enzyme-linked immunosorben assay (Immunodiagonstic System Ltd., Boldon, UK), and absorbance was read using ELISA reader (Thermo, USA) at $450 \mathrm{~nm}$.

\section{Immunohistochemical analysis}

Collagen I and Collagen III was identified in paraffinembedded sections of the lung tissue by immunohistochemical staining with anti-Collagen I antibody or 
anti-Collagen III antibody (Abcam, Cambridge, UK) overnight at $4{ }^{\circ} \mathrm{C}$ at a concentration of $1: 100$ following by standard biotin-streptavidin-peroxidase immunostaining using a streptavidin-peroxidase kit (Beijing Zhongshan Goldenbridge Biotechnology, Beijing, China) following the instructions provided by the manufacturer. Staining was completed by incubation with diaminobenzidine chromogen solution at room temperature. All measurements were performed with the Nikon ECLIPSE 80i biomicroscope and NIS-Elements BR 3.2 image analysis system (Nikon, Japanese). Three random images within a lung sample from 3 transverse sections were taken, and further analyzed by using zoomed-in field at $400 \times$ magnification. We measured the integral optical density (IOD) and the area of Collagen I or Collagen III-positive cells under each examined field, and calculated the average number as the final result of this sample.

\section{Quantitative real-time PCR (qPCR)}

Total RNA was isolated from the lung tissue using TRIzol reagent (Invitrogen, CA, USA) according to the manufacturer's recommendations. Total RNA $(2 \mu \mathrm{g})$ was reverse-transcribed using the Superscript First Strand synthesis system (Invitrogen, CA, USA) to generate complementary DNA (cDNA). The qPCR amplification was performed using the SYBR-green detection of PCR products in real time with an ABI-7500 Sequence Detection System (Applied Biosystems, Foster City, CA, USA). The primers used in the qPCR analysis are presented in Table 1. The PCR program was performed for 40 cycles with each cycle consisting of $30 \mathrm{~s}$ of denaturation at $95^{\circ} \mathrm{C}$, $3 \mathrm{~s}$ of annealing at $95^{\circ} \mathrm{C}$, and $30 \mathrm{~s}$ of extension at $60^{\circ} \mathrm{C}$. Gene expression was quantified by means of the comparative $\mathrm{Ct}$ method $\left({ }^{\Delta \Delta} \mathrm{Ct}\right)$ and the relative quantification (RQ) was calculated as $2^{-\Delta \Delta C t}$. Relative mRNA levels of TGF$\beta 1$, TGF- $\beta 2$, Smad2, Smad3 and Smad7 were examined and normalized to $\beta$-actin mRNA expression in each sample. The melting curves for each PCR reaction were generated to ensure the purity of the amplification product. A no-template negative control was included in each experiment.

Table 1 Primers used for $\mathrm{qPCR}$ analysis

\begin{tabular}{|c|c|c|}
\hline Primer & Forward primer & Reverse primer \\
\hline TGF- $\beta 1$ & TGAGTGGCTGTCTTITGACG & TGGGACTGATCCCATTGATT \\
\hline TGF- $\beta 2$ & GCGAGCGAAGCGACGAGGAG & TGGGCGGGATGGCATCAAGGTA \\
\hline $\operatorname{mad} 2$ & CGATGCTCAAGCATGTCCTA & CGCTCTGGGTITTGACTAGC \\
\hline Smad3 & TITAGCATTCTGCCGCTTTT & TGCCCCAGTTTAACCAAGTC \\
\hline Smad7 & CCAACTGCAGACTGTCCAGA & CAGGCTCCAGAAGAAGTTGG \\
\hline$\beta$-actin & AGCCATGTACGTAGCCATCC & ACCCTCATAGATGGGCACAG \\
\hline
\end{tabular}

\section{Statistical analysis}

Results of all measurements were presented as means \pm standard deviation (SD). The data analysis was performed using the SPSS 13.0 (SPSS Inc., Chicago, USA). All of the data were tested for normality using the Kolmogorov-Smirnov test, and passed. A one-way analysis of variance (ANOVA) was performed to determine whether there were statistically significant differences $(P<0.05)$ among the experimental groups. The Duncan's multiple range post-hoc test was used for comparisons between individual groups and to determine which means differed statistically significantly $(P<0.05)$.

\section{Results}

\section{Effects of budesonide and the herbal extracts on airway} remodeling in H\&E sections

To determine whether budesonide and the herbal extracts were involved in the development of airway remodeling, we evaluated the peribronchial cellular infiltration and airway smooth muscle thickness in all experimental rats. Representative sections of each group were stained with H\&E (Fig. 1d). As shown in Fig. 1d, no inflammation, mucosal edema and epithelial lesions were observed in the control group, whereas OVAinduced asthma rats developed severe inflammation, mucosal edema and epithelial lesions, which included interstitial infiltrates and a large number of lymphocyte and eosinophil infiltration. After treating with budesonide, the herbal extracts or the Co-administration, mild to moderate inflammation, mucosal edema and epithelial lesions were observed. According to results of the morphometry of H\&E sections, we calculated the ratios of $\mathrm{Wat} / \mathrm{Pbm}$ and $\mathrm{Wam} / \mathrm{Pbm}$ to evaluate airway remodeling. Figure $1 \mathrm{~b}$ showed that the OVA-induced asthma rats presented thicker airway walls than the normal control group $(P<0.01)$ after correction for airway basement perimeter $(\mathrm{Wat} / \mathrm{Pbm})$. Budesonide and Coadministration groups could effectively reduce airway wall thickening (Wat/Pbm; $P<0.05$ and $P<0.01$, respectively). For Wam/Pbm (Fig. 1c), the asthma model group had an increased smooth muscle layer compared with the normal control group $(P<0.01)$, and only the Co-administration group could reduce myocyte hyperplasia $(P<0.05)$. There were no significant differences in Wat/Pbm and Wam/Pbm between the groups of Budesonide and Extracts.

\section{Effects of budesonide and the herbal extracts on collagen deposition and goblet cell hyperplasia}

Mucus hypersecretion and collagen hyperplasia, which are the main pathological features in asthma and contributes significantly to airflow limitation, are accompanied by Collagen deposition and goblet cell hyperplasia. Collagen deposition within the rat airway wall was 


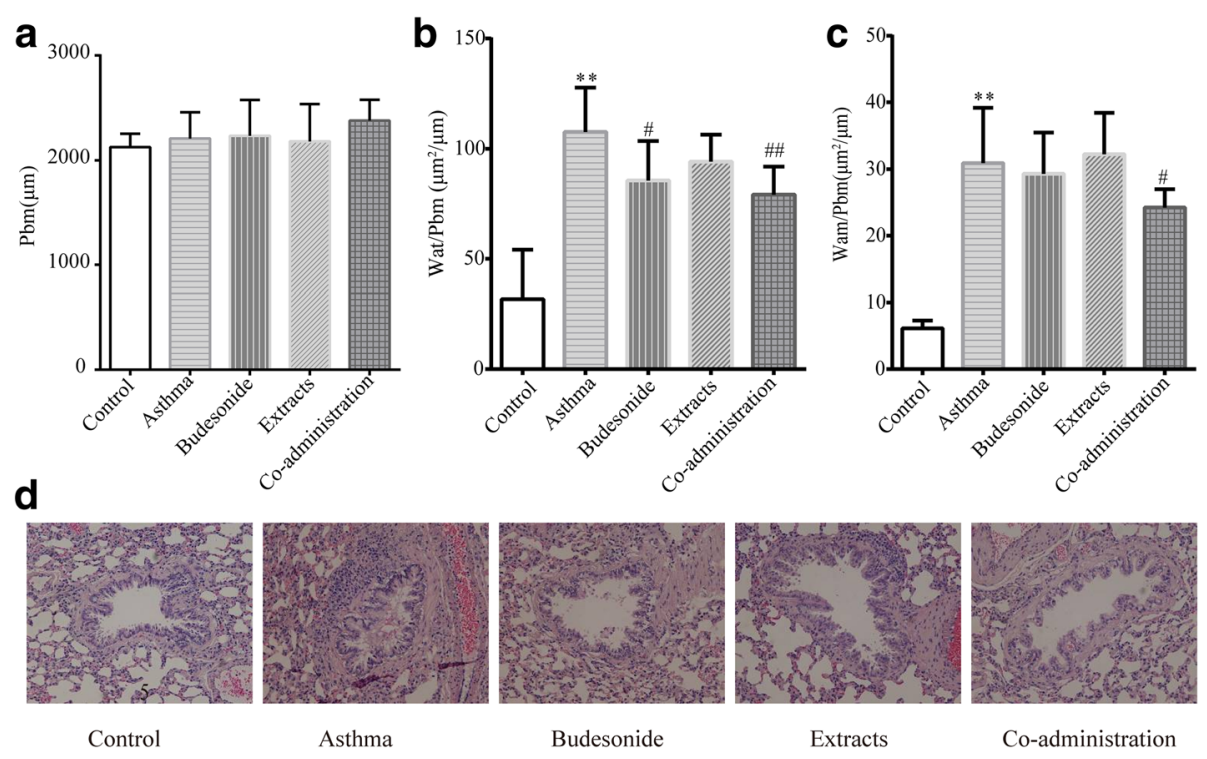

Fig. 1 Effects of budesonide and the herbal extracts on airway remodeling. Pbm (a), Wat/Pbm (b), and Wam/Pbm (c) in H\&E-stained lung sections. (d) Representative photomicrographs of H\&E-stained lung sections from each group ( $\times 400)$; Mean $\pm \mathrm{SD}, n=10$. ${ }^{* *} P<0.01$ compared to normal control group; ${ }^{\#} P<0.05$ and ${ }^{\#} P<0.01$ compared to asthma model group

performed by the Masson's trichrome stainning. Figure 2a showed that the area of peribronchial collagen fiber was significantly greater in the asthma model group than that in the normal control group $(P<0.01)$. Only the Coadministration group could significantly decrease the area of collagen deposition, compared with the asthma group $(P<0.05)$. PAS-stained lung sections were performed to evaluate goblet cell hyperplasia in the airway epithelium, which is a cardinal feature of bronchial asthma. As shown in Fig. 2e, goblet cell hyperplasia was observed in the asthma model group, but not in the control group. The number and area of goblet cells in the epithelium greatly increased following repeated OVA-challenge $(P<0.01)$, and showed the hypertrophic features. Compared with the asthma model group, a significant decrease was noticed in airway secretion in all three treatment groups (all $P<0.01$ ), which indicated that budesonide and herbal extracts markedly reduced goblet cell hyperplasia in airways. There was a significant difference in goblet cell number between the budesonide group and the co-administration group $(P<0.05)$. These data indicated that the combination of budesonide and herbal extracts had a better effect in reducing goblet cell hyperplasia.

\section{Effects of budesonide and the herbal extracts on airway inflammation}

To investigate the function of the co-administration of budesonide and the herbal extracts in OVA-induced inflammatory infiltration in the airways, we examined the cell count in BALF cytospin. As shown in Fig. 3d and e, the number of lymphocytes and eosinophils increased in the BALF of the OVA-induced asthmatic rats compared with those in the normal control group. Only the treatment with the co-adminstration of budesonide and the herbal extracts significantly inhibited the increase in the number of lymphocytes, and each group of drug administration significantly decreased the number of eosinophils, compared with the asthma model group.

IL-4, IL-5 and IgE were closely related to airway variability inflammation. As shown in Fig. 3a, b and c, the content of serum IL-4, IL-5 and IgE were significantly increased after modeling $(P<0.01)$. Compared with the asthma model group, the serum levels of IL-4, IL-5 and IgE in each group of drug administration was remarkably decreased $(P<0.01)$. In addition, there was a significant difference in the level of IL-5 between the groups of budesonide and co-administration.

\section{Effects of budesonide and the herbal extracts on collagen I and collagen III}

Collagen I and collagen III are the most important components of extracellular matrix during collagen deposition in airway remodeling. Immunohistochemical staining results showed that the immunostaining area and IOD of collagen I and collagen III in the asthma group were significantly greater than those in the control group $(P<0.01$; Fig. 4). Both the extracts group and coadministration group significantly reduced the area and IOD of collagen I and collagen III compared with those in the asthma model group $(P<0.05$ or $P<0.01)$. There was no significant difference of collagen I expression between the asthma group and the budesonide group. 

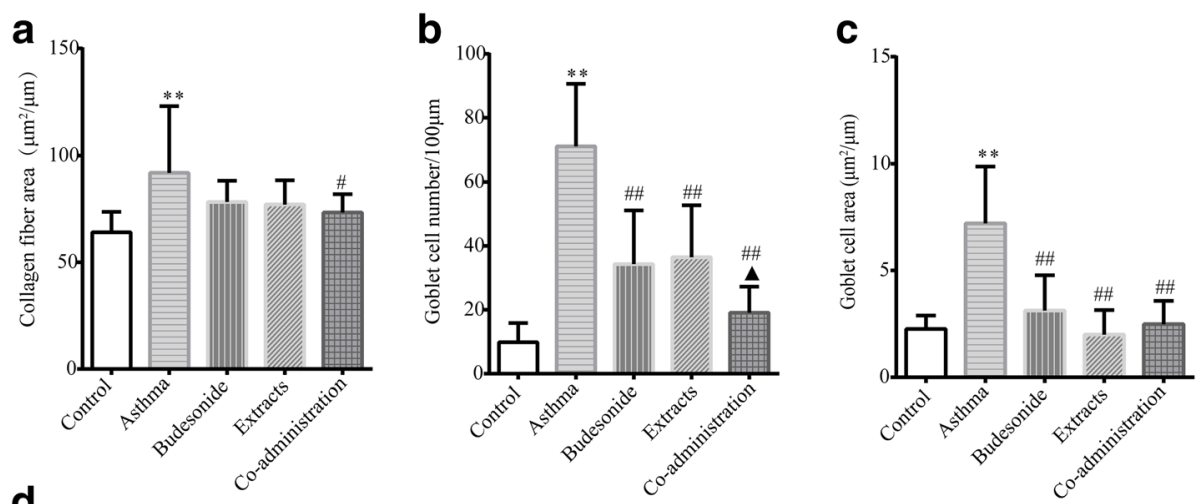

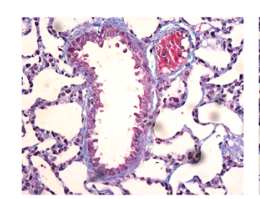

Control

E

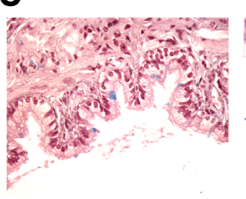

Control

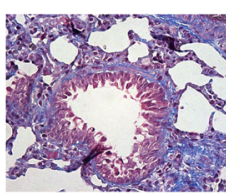

Asthma

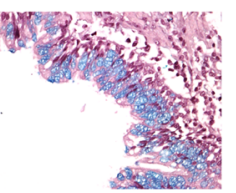

Asthma

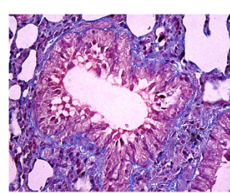

Budesonide

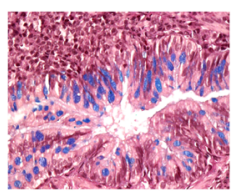

Budesonide

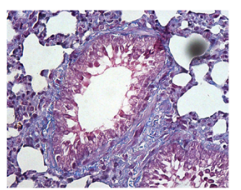

Extracts

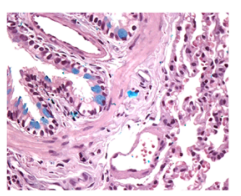

Extracts

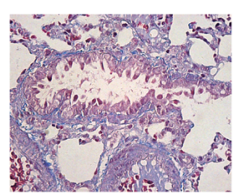

Co-administration

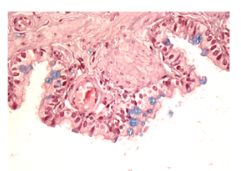

Co-administration

Fig. 2 Effects of budesonide and the herbal extracts on collagen deposition and goblet cell hyperplasia. (a) Collagen fiber area in Masson's trichrome-stained sections. (b) The number of the goblet cells in PAS-stained lung sections. (c) The area of the goblet cells in PAS-stained lung sections. (d) Representative photomicrographs of Masson's trichrome-stained sections from each group $(\times 400)$. (e) Representative photomicrographs of PAS-stained lung sections from each group ( $\times 400)$. Mean $\pm S D, n=10$. ${ }^{* *} P<0.01$ compared to normal control group; ${ }^{\#} P<0.05$ and ${ }^{\# \#} P<0.01$ compared to asthma model group; $\mathbf{\Delta} P 0.05$ compared to budesonide group

The budesonide group only decreased collagen III IOD $(P<0.05)$. There was a significant difference in collagen III IOD between the budesonide group and the coadministration group $(P<0.01)$, indicating that the combination of budesonide and the herbal extracts had a better effect in reducing collagen III hyperplasia.

\section{Effects of budesonide and the herbal extracts on TGF- $\beta$ / Smads}

It is essential to understand the mechanism of coadministration of budesonide with the herbal extracts for using in clinical development, effectively. In view of recent studies show that TGF- $\beta 1$ and TGF- $\beta 2$ play important roles in promoting the structural changes of airway remodeling in asthma [37-39]. The mRNA levels of TGF- $\beta 1$ and TGF- $\beta 2$ in the five groups were determined by qPCR. Our data showed that the mRNA expressions of TGF- $\beta 1$ and TGF- $\beta 2$ in the asthma group were significantly increased compared with the normal control group $(P<0.05$ and $P<0.01)$. TGF- $\beta 1$ mRNA expression only in the Co-administration group and TGF- $\beta 2$ mRNA expression in all the three treatment groups were decreased compared with that in the asthma group
$(P<0.05$ or $P<0.01)$. In addition, there was a significant difference in TGF- $\beta 1$ or TGF- $\beta 2$ mRNA expression between the budesonide group and the co-administration group $(P<0.01)$ (Fig. 5a and b).

In order to investigate the expression of active TGF- $\beta$ signaling in situ, we examined the expression of the intracellular effectors, Smads. An increase in the mRNA expression of Smad2/3 and a decrease in that of Smad7 were observed during prolonged allergen challenge (all $P<0.01$ ). All of the treatment groups could considerably decreased Smad2 mRNA expression $(P<0.05$ or $P<0.01)$. Only coadministration group could considerably altered Smad3 and Smad7 mRNA expression (both $P<0.05$ ). There was a significant difference in Smad2 mRNA expression between the budesonide group and the co-administration group $(P<0.05)$. And there was a significant difference in Smad3 mRNA expression among rats treated with the herbal extracts and the co-administration of budesonide and the herbal extracts $(P<0.05)$ (Fig. $5 \mathrm{c} \sim$ e).

\section{Discussion}

Asthma is a chronic inflammatory airway disease that is associated with airway remodeling and airway 

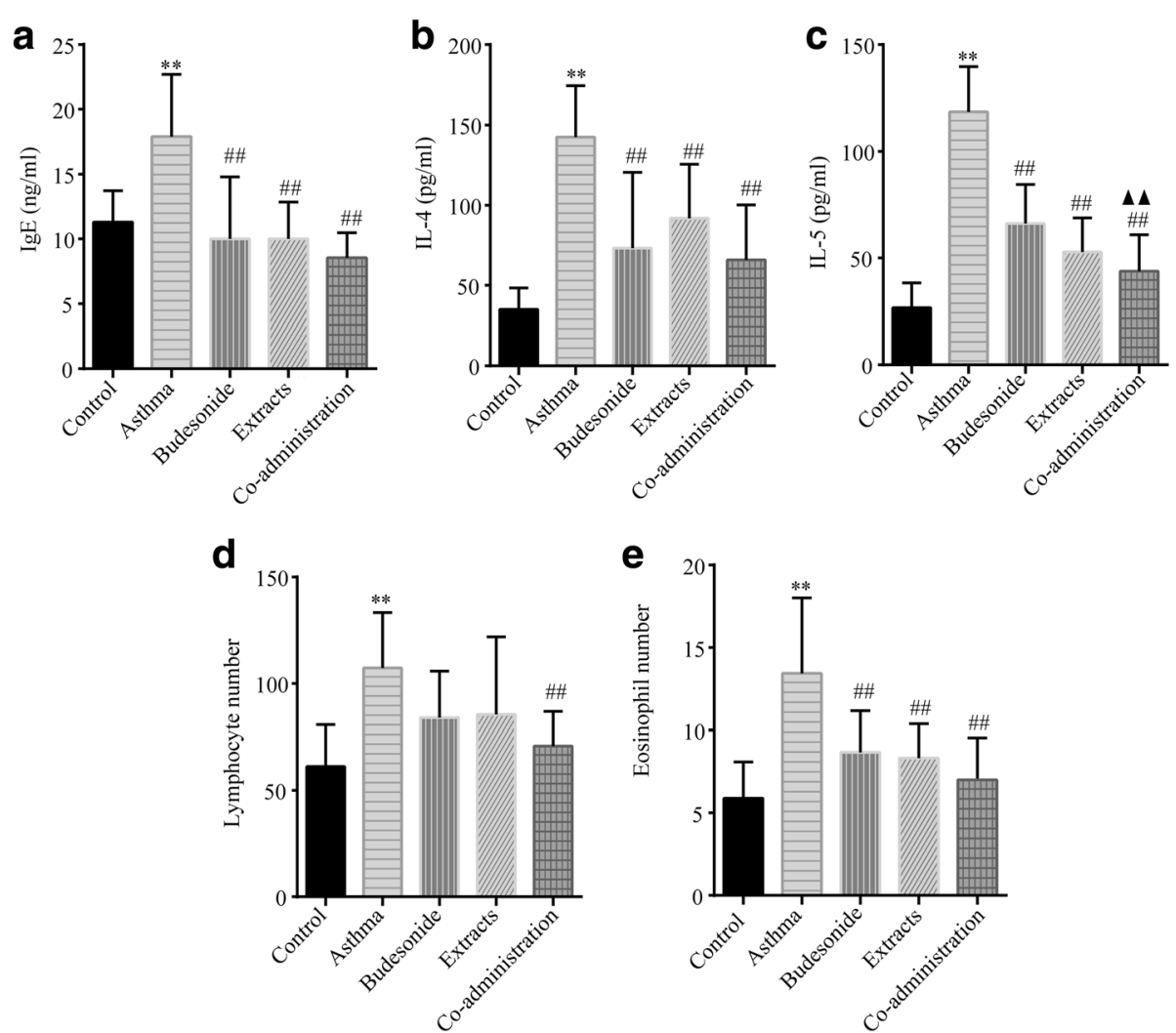

Fig. 3 Effects of budesonide and the herbal extracts on airway inflammation. The serum levels of IL-4 (a), IL-5 (b) and IgE (c) were assayed by ELISA. Lymphocytes (d) and eosinophils (e) were count on BLAF cytospin by Wright-Giemsa staining. The serum levels of IL-4, IL-5 and IgE and the number of lymphocytes and eosinophils in the OVA-induced asthmatic rats were significantly increased compared with those in the normal control group. Mean $\pm \mathrm{SD}, n=9$. ${ }^{* *} P<0.01$ compared to normal control group; ${ }^{\# \#} P<0.01$ compared to asthma model group; ${ }^{\boldsymbol{\Delta}} P<0.01$ compared to budesonide group

hyperresponsiveness (AHR). Airway inflammation is considered to be the basic pathological change and one of the key pathological mechanisms of repeated attacks of asthma [40,41], characterized by the infiltration of eosinophils and dominant in Th2 cytokines $[4,5]$. IgE is a critical factor for the development of AHR and airway inflammation in asthmatics [42]. And the total IgE level is related to new-onset asthma and the severity of asthma attacks [43, 44]. Allergenspecific Th2 cells are key players in induction and maintenance of allergic asthma. IL-4 is commonly accepted to play a crucial role in driving the differentiation of $\mathrm{CD}^{+}{ }^{+}$Th precursors into Th2-like cells [45]. Apart from determining IgE synthesis by B lymphocytes, IL-4 increases the expression of an inducible form of the low-affinity receptor for IgE (FceRII/ CD23) on B cells [46]. IL-5, another Th2-like cytokines, has profound effects on eosinophil differentiation, activation and survival [47]. The results of the present study show that the number of lymphocytes and eosinophils in BALF and the levels of IL-4, IL-5 and IgE in serum significantly increased in the
OVA-induced asthma model rat, while the extracts of $\mathrm{HE}$ and FLL, budesonide and the combination of the extracts of HE and FLL with budesonide significantly decreased the levels of IL-4, IL-5 and IgE in serum and the number of eosinophils in BALF. The combined administrations of budesonide with the herbal extracts had better effects on serum IL-5 level than budesonide administration. These results suggest that the combined administrations of budesonide with the herbal extracts might have synergistic effects on airway inflammation.

Airway remodeling is an irreversible airway hyperplasia process that contributes to AHR and irreversible airflow limitation. Airway remodeling is characterized by the increased deposition of collagen in the subepithelial basement membrane region and submucosal layers, smooth muscle hypertrophy and hyperplasia, fibroblast hyperplasia, epithelial metaplasia and goblet cell proliferation $[48,49]$. The poor response to treatment observed in patients with refractory asthma may be a consequence of ongoing airway remodeling that result in fixed airway obstruction [50]. We observed that all of the three 

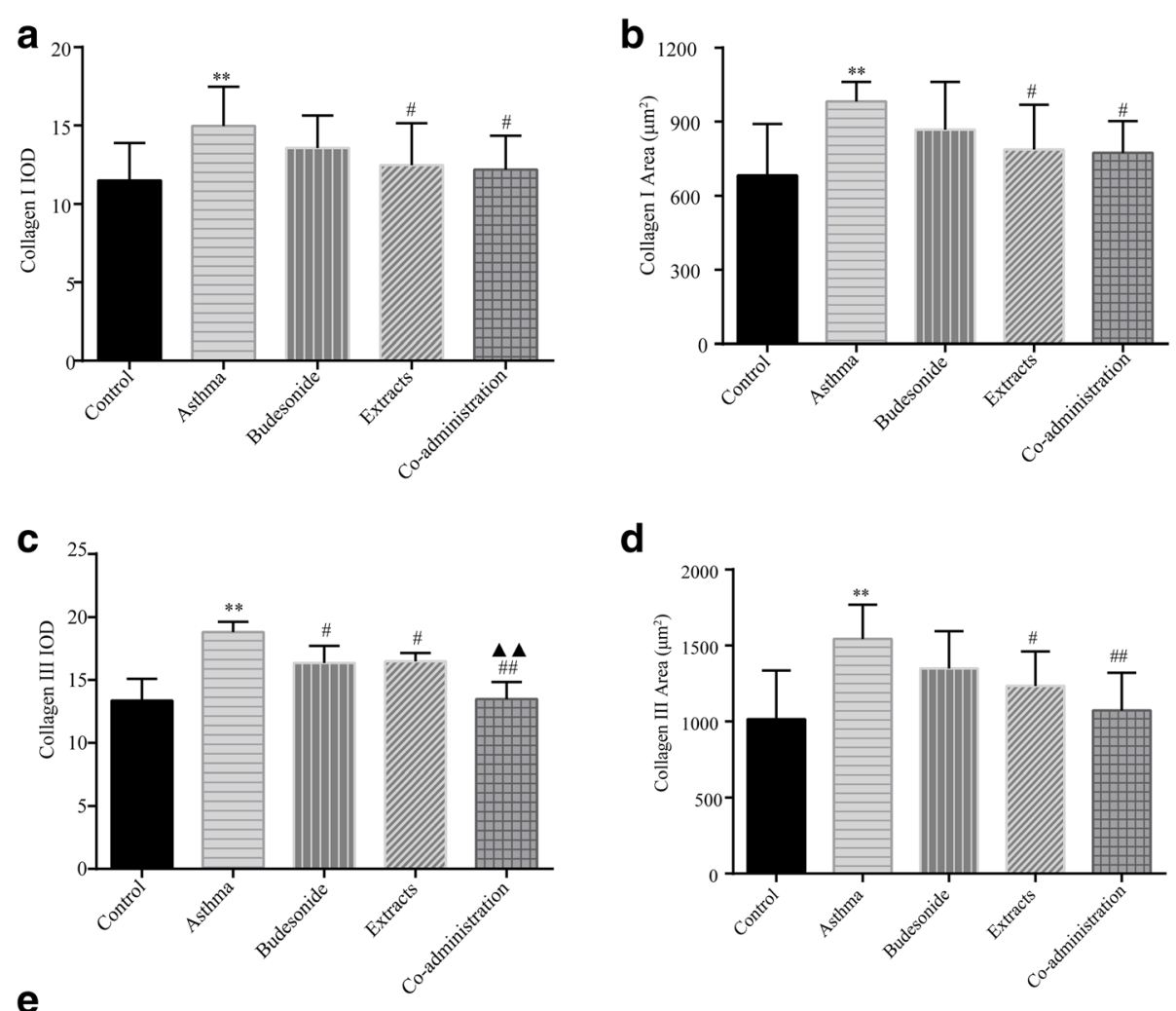

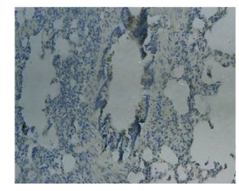

Control

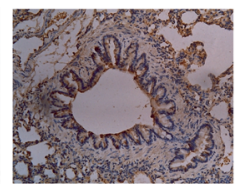

Asthma

f

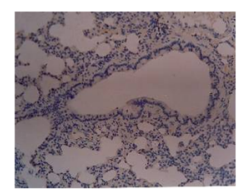

Control

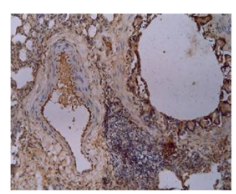

Asthma

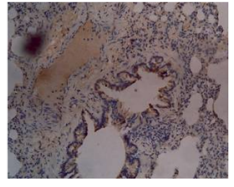

Budesonide

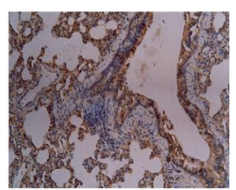

Budesonide

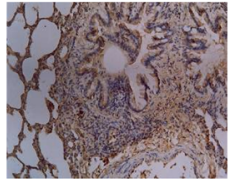

Extracts

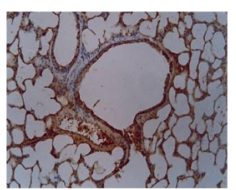

Extracts

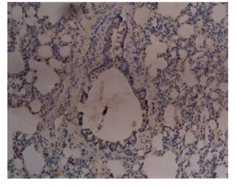

Co-administration

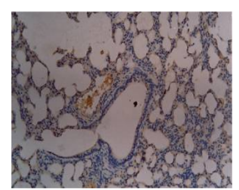

Co-administration

Fig. 4 Effects of budesonide and the herbal extracts on expression of collagen I and collagen I. Expression of the two types of collagen in lung tissue was determined by immunohistochemical staining. (a) IOD of collagen I. (b) Positive area of collagen I. (c) IOD of collagen III. (d) Positive area of collagen III. (e) Representative photomicrographs of collagen I from immunohistochemical-stained lung sections ( $\times 400)$. (f) Representative photomicrographs of collagen III from immunohistochemical-stained lung sections ( $\times 400)$. Mean $\pm \mathrm{SD}, n=8 .{ }^{* *} P<0.01$ compared to normal control group; ${ }^{\#} P<0.05$ and ${ }^{\# \#} P<0.01$ compared to asthma model group; $\mathbf{\Delta} \boldsymbol{\Delta} P<0.01$ compared to budesonide group

treatment groups could reduce goblet cell hyperplasia according to the morphometry of PAS-stained lung sections. Only the combination of the extracts of HE and FLL with budesonide significantly decreased collagen deposition according to the morphometry of Masson'sstained lung sections; meantime inhibited the thickening of airway wall and smooth muscle layer and epithelial hyperplasia according to the morphometry of H\&Estained lung sections. These results suggest that the coadministrations of budesonide with the herbal extracts might have a better synergistic effect on the pathological changes of airway remodeling, as compared to the single use of budesonide or the herbal extracts.

To further confirm that the sub-epithelial area of blue staining measured from Masson's-stained sections truly reflected an increase in collagen deposition, we also immunostained sections for collagen I and collagen III. Types I and III collagens represent greater than $95 \%$ of total lung collagen [51, 52]. Compared with the total blue Masson's-stained area, the proportional area of 

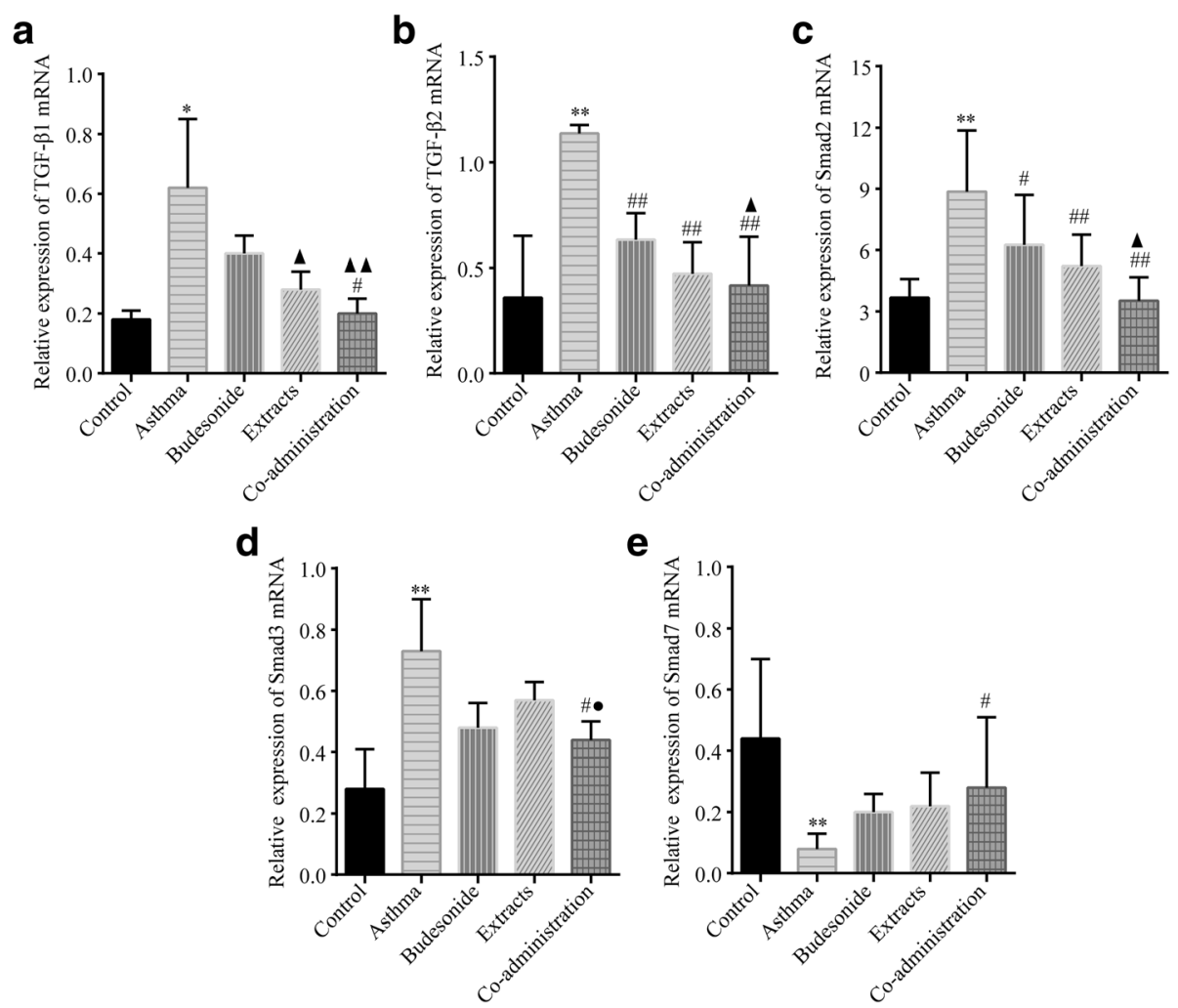

Fig. 5 Effects of budesonide and the herbal extracts on mRNA expression of TGF- $\beta / S$ mads. The mRNA expressions of TGF- $\beta 1$ (a), TGF- $\beta 2$ (b), Smad2 (c), Smad3 (d) and Smad7 (e) in lung tissue were determined by qPCR with $\beta$-actin as an internal control. In the asthmatic rats, the mRNA expressions of TGF- $\beta 1$, TGF- $\beta 2$, Smad2 and Smad3 were significantly higher, while that of Smad7 was significantly lower than those in the normal group. Mean \pm SD, $n=7 .{ }^{*} P<0.05$ and ${ }^{* *} P<0.01$ compared to normal control group; ${ }^{\#} P<0.05$ and ${ }^{\#} P<0.01$ compared to asthma model group; ${ }^{*} P<0.05$ and $\boldsymbol{\Delta} \boldsymbol{\Delta} P<0.01$ compared to budesonide group; $\bullet P<0.05$ compared to extracts group

immunostained collagen I and collagen III is consistent with the distribution of collagen types in the lung. Quantified analysis of immunohistochemical sections revealed that all of the three treatment groups reduced IOD of collagen III, but the decrease in the coadministration group is significant, as compared to the budesonide group. The extracts group and the co-administration group could depress collagen I IOD and positive area of collagen I and Collagen III. Our study indicates that the co-administration of budesonide and the herbal extracts could prevent and inhibit collagen aggregation of airway remodeling process in allergic airway diseases.

Recently, the TGF- $\beta /$ Smads signaling pathway was found to be one of the important mechanisms involved in the development of airway remodeling in asthma $[53,54]$. Three isoforms of TGF- $\beta$ are found in mammals, two of which (TGF- $\beta 1$ and TGF- $\beta 2$ ) appear to have critical roles in bronchial asthma. TGF- $\beta 1$ (most abundant isoform, characteristically associated with endothelial, hematopoietic and connective tissue cells) promotes the expression of profibrotic mediators in various cell types. Furthermore, it is a key component in inducing the epithelial-mesenchymal transition (EMT). In vitro studies have shown that TGF- $\beta 1$ stimulates the proliferation of mesenchymal cells; moreover, it induces the differentiation of fibroblasts into myofibroblasts to synthesize extracellular matrix (ECM) proteins. Thus, TGF- $\beta 1$ has the ability to elicit many of the structural alterations of airway remodeling $[55,56]$. TGF- $\beta 2$ (primarily synthesized by airway epithelial cells and neuronal cells) enhances fibroblast activity in the context of inflammatory remodeling in respiratory epithelium. TGF- $\beta 2$ is secreted in response to epithelial injury [57]. Furthermore, TGF- $\beta 2$ stimulates collagen synthesis in fibroblasts and is thought to be an important mediator of subepithelial fibrosis. Epithelial-derived TGF- 32 can drive collagen synthesis in subepithelial fibroblasts $[58,59]$. As important members of the TGF- $\beta$ signal transduction system, Smads are the group of intracellular proteins that are critical for transmitting the TGF- $\beta$ signals from the cell surface to the nucleus to promote transcription of target genes [60]. Among Smads, Smad2 and Smad3 form a heterocomplex with Smad4, and transfer into the cellular nucleus activating DNA transcription to 
regulate the target gene expression. On the other hand, Smad7 can block the transcription induced by TGF- $\beta$ through inhibiting its signaling pathway [61, 62]. In our study, we found that treatment with the combinations of the herbal extracts with budesonide reduced the mRNA expression of TGF- $\beta 1$, TGF- $\beta 2$, Smad2 and Smad3, while increased Smad7 mRNA expressions. It was reported that there was a link to the expression of TGF- $\beta$ and the deposition of both collagen I and III in bronchial biopsies, and the expression of both collagen I and III were significantly greater in the subjects with more severe asthma. This effect was not modified following treatment with corticosteroids suggesting that remodeling that had already occurred was not reversible by corticosteroids $[63,64]$. So we deduced that the decrease of TGF- $\beta 1$ and TGF- $\beta 2$ levels and modulation of the activity of the TGF- $\beta$ signaling pathway might be a possible mechanism by which the co-administration of budesonide with the herbal extracts inhibits airway remodeling in asthma.

The detailed compositions of both HE and FLL have been previously reported, and mainly include flavonoids, iridoid glycosides and alkaloids, all of which may contribute to the anti-inflammation and anti-remodeling effects of the two herbs. Icariin, identified as the major active ingredient of $\mathrm{HE}$, could exert the antiinflammatory and anti-tumor functions $[65,66]$. Oleanolic acids are the major pharmacologically active components in FLL with anticancer activity, antioxidant activity and immunomodulating effect [67-69]. These results suggest that the anti-inflammatory and anti-remodeling effects of the extracts of HE and FLL may be the result of cooperative actions among multiple compounds with their multiple components working together to common affects. The exact roles of the different active components when used for the treatment of asthma remain to be further investigated.

\section{Conclusions}

In summary, our study demonstrated that inhaled GC (budesonide) combined with the extracts of HE and FLL has a better synergistic effect on the airway remodeling in asthma rats, compared with the single use of budesonide or the herbal extracts. It was deduced that the mechanisms of anti-remodeling in asthma airway involve in inhibiting the mRNA expression of TGF- $\beta 1$ and TGF$\beta 2$ as well as modulation of TGF- $\beta$ signaling in the lung tissue. Additional studies exploring the mechanism of the systemic therapy are needed.

\section{Abbreviations}

AHR: Airway hyperresponsiveness; ANOVA: One-way analysis of variance; BALF: Bronchoalveolar lavage fluid; CDNA: Complementary DNA; ECM: Extracellular matrix; EMT: Epithelial-mesenchymal transition; FLL: Fructus Ligustri Lucidi; GC: Glucocorticoid; GR: Glucocorticoids receptor; H\&E: Hematoxylin and eosin; HE: Herba Epimedii; HPA: Hypothalamic-pituitary-adrenal; IL: Interleukin; IOD: Integral optical density; ip: Intraperitoneal; OVA: Ovalbumin; PAS: Periodic-acid schiff; Pbm: Perimeter of basement membrane; qPCR: Quantitative Real-Time PCR; RQ: Relative quantification; SD: Standard deviation; TCM: Traditional Chinese Medicine; TGF: Transforming growth factor; Th: Helper T cells; Wam: Area of smooth muscle; Wat: Total area of airway wall

\section{Acknowledgments \\ Shizeng Li and Guiru Zhu conducted the conception and design of the study, acquisition and interpretation of data and final approval of the version to be published.}

\section{Funding}

This work was supported by the grants from the National Natural Science Foundation of China (no. 81673993 and no.81373814), Scientific Research Common Program of Beijing Municipal Commission of Education (no. KM201510025012), the Importation and Development of High-Caliber Talents Project of Beijing Municipal Institutions (no. CIT\&TCD201504097), and Planned Project on Beijing Traditional Chinese Medicine inheritance of $3+3$ programmer of Beijing Chinese Medicine Administration Bureau (no.2012-SZ-C-42).

\section{Availability of data and materials}

The authors declare that all the data and materials described in our publication are freely available when they may be reasonably requested by others.

\section{Authors' contributions}

RHL and XFT performed the rat experiments and drafted the manuscript. $X F T, H L N, X X L, Y Y, H T S$ and $X W Y$ were mainly involved in the rat experiments and the index detection. XJW and LPX were heavily involved in the experimental design and the scientific correction of the draft manuscript. All authors have read and approved the final manuscript.

\section{Ethics approval}

The experimental protocol was approved by the Animal Care Committee of Capital Medical University, Beijing, China (No. AEEl-2014-045).

\section{Consent for publication}

The authors declare that they consent to publish this manuscript.

\section{Competing interests}

The authors declare that they have no competing interests.

\section{Publisher's Note}

Springer Nature remains neutral with regard to jurisdictional claims in published maps and institutional affiliations.

Received: 29 August 2016 Accepted: 24 July 2017

Published online: 01 August 2017

\section{References}

1. Braman SS. The global burden of asthma. Chest. 2006;130(1 Suppl):4-12.

2. Steppuhn H, Langen U, Scheidt-Nave C, Keil T. Major Comorbid conditions in asthma and association with asthma-related hospitalizations and emergency department admissions in adults: results from the German national health telephone interview survey (GEDA) 2010. BMC Pulm Med. 2013;13:46.

3. Zhu M, Liang Z, Wang T, Chen R, Wang G, Ji Y. Th1/Th2/Th17 cells imbalance in patients with asthma with and without psychological symptoms. Allergy Asthma Proc. 2016;37(2):148-56.

4. Jacobsen EA, Lee NA, Lee JJ. Re-defining the unique roles for eosinophils in allergic respiratory inflammation. Clin Exp Allergy. 2014;44(9):1119-36.

5. Subbarao P, Mandhane PJ, Sears MR. Asthma: epidemiology, etiology and risk factors. CMAJ. 2009;181(9):E181-90.

6. Hassan M, Jo T, Risse PA, Tolloczko B, Lemie're C, Olivenstein R, Hamid Q, Martin JG. Airway smooth muscle remodeling is a dynamic process in severe long-standing asthma. J Allergy Clin Immunol. 2010;125(5):1037-45.

7. Trifilieff A, El-Hashim A, Bertrand C. Time course of inflammatory and remodeling events in a murine model of asthma: effect of steroid treatment. Am J Physiol Lung Cell Mol Physiol. 2000;279(6):L1120-8.

8. Reddel HK, Bateman ED, Becker A, Boulet LP, Cruz AA, Drazen JM, Haahtela T, Hurd SS, Inoue H, de Jongste JC, Lemanske RF Jr, Levy ML, O'Byrne PM, Paggiaro P, Pedersen SE, Pizzichini E, Soto-Quiroz M, Szefler SJ, Wong GW, FitzGerald JM. A summary of the new GINA strategy: a roadmap to asthma control. Eur Respir J. 2015;46(3):622-39. 
9. Boulet LP, Hernandez P, Devlin H, Freeman MA, Gupta S. Asthma and chronic obstructive pulmonary disease guideline implementation: lessons learned on recruitment of primary care physicians to a knowledge translation study. Can Respir J. 2013;20(4):275-80.

10. Barnes PJ. Glucocorticosteroids: current and future directions. Br J Pharmacol. 2011;163(1):29-43.

11. Ducharme FM. Inhaled glucocorticoids versus leukotriene receptor antagonists as single agent asthma treatment: systematic review of current evidence. BMJ. 2003;326(7390):621.

12. Irwin RS, Richardson ND. Side effects with inhaled corticosteroids: the physician's perception. Chest. 2006;130(S1):41S-53S.

13. Phillip M, Aviram M, Leiberman E, Zadik Z, Giat Y, Levy J, Tal A. Integrating plasma cortisol concentration in children with asthma receiving long-term inhaled corticosteroids. Pediatr Pulmonol. 1992;12(2):84-9.

14. Zöllner EW, Lombard C, Galal U, Hough S, Irusen E, Weinberg E. Hypothalamicpituitary-adrenal axis suppression in asthmatic children on inhaled and nasal corticosteroids-more common than expected? J Pediatr Endocrinol Metab. 2011;24(7-8):529-34.

15. Heuck C, Wolthers OD, Hansen M, Kollerup G. Short-term growth and collagen turnover in asthmatic adolescents treated with the inhaled glucocorticoid budesonide. Steroids. 1997;62(10):659-64

16. Li XM, Brown L. Efficacy and mechanisms of action of traditional Chinese medicines for treating asthma and allergy. J Allergy Clin Immunol. 2009: 123(2):297-306.

17. Abdureyim S, Amat N, Umar A, Upur H, Berke B, Moore N. Anti-inflammatory, immunomodulatory, and heme oxygenase-1 inhibitory activities of Ravan Napas, a formulation of Uighur traditional medicine, in a rat model of allergic asthma. Evid Based Complement Alternat Med. 2011;2011:725926.

18. Shen ZY, Hu GR, Xu DS, Chen WH, Zhang $\sqcup$. The mechanism of tonify kidney to prevent allergic and non-allergic asthma. Zhongguo Zhong Xi Yi Jie He Za Zhi. 1989;9(20):82-4.

19. Zhao FD, Dong JC, Xie JY. Effects of Chinese herbs for replenishing shen and strengthening qi on some indexes of neuro-endocrino-immune network in asthmatic rats. Zhongguo Zhong Xi Yi Jie He Za Zhi. 2007;27(8):715-9.

20. Gong ZH, Dong JC, Xie JY. Regulatory function of Shen-reinforcing and qisupplementing drugs on hypothalamic-pituitary-adrenal axis and interleukin-6 dysfunction in asthmatic rats. Zhongguo Zhong Xi Yi Jie He Za Zhi. 2008;28(4): 348-51.

21. Zhao L, Wu H, Qiu M, Sun W, Wei R, Zheng X, Yang Y, Xin X, Zou H, Chen T, Liu J, Lu L, Su J, Ma C, Zhao A, Jia W. Metabolic signatures of kidney Yang deficiency syndrome and protective effects of two herbal extracts in rats using GC/TOF MS. Evid Based Complement Alternat Med. 2013;2013:540957.

22. Li B, Luo QL, Nurahmat M, Jin HL, Du YJ, Wu X, Lv YB, Sun J, Abduwaki M, Gong WY, Dong JC. Establishment and comparison of combining disease and syndrome model of asthma with "kidney yang deficiency" and "abnormal savda". Evid Based Complement Alternat Med. 2013;2013:658364.

23. Huang Q, Liu RH, Guo X. An experimental study on the adjustment of disorder of hypothalamic-pituitary-adrenal axis by Peibenfang in asthmatic rat. Sh J Prev Med. 2006;17(12):568-9.

24. Liu RH, Guo X. Experimental study of the adjustment of Peibenfang on the functional route of endogenous glucocorticoid (GC) in asthma rats. Lishizhen Medicine and Materia Medica research. 2007;18(11):2765-7.

25. Yang $Y$, Nian $H L$, Tang XF, Wang XJ, Liu RH. Effects of the combined Herba Epimedii and Fructus Ligustri Lucidi on bone turnover and TGF- $\beta 1 / S m a d s$ pathway in GIOP rats. J Ethnopharmacol. 2017;201:91-9.

26. Kang X, Liu RH, Wang XJ. Advances on studies of snti-osteoporosis spplications and mechanisms by Herba Epimedii and Fructus Ligustri Lucidi. Chinese Journal of Experimental Traditional Medical Formulae. 2012;18(21):331-4.

27. Liu R, Xu L, Wang X, Yang J, Wang P, Pan L. Experimental study on effect of combination of Epimedii folium/Ligustri Lucidi Fructus and dexamethasone on asthmatic rats. Zhongguo Zhong Yao Za Zhi. 2012;37(10):1497-599.

28. Liu R, Zheng J, Yuan Y, Guo X. Adjustment effects of Herba epimedii, Fructus ligustrilucidi on NO/ET, HPA axis in asthmatic rats. Zhongguo Zhong Yao Za Zhi. 2010;35(12):1590-3.

29. Liu R, Wang X, Zhang W, Yuang Y, Wang P. Effects of combination of Herba Epimedii, Ligustrum Lucidum and dexamethasone on HPA axis and GR of asthmatic model in rats. Zhonghua Zhong Yi Yao Za Zhi. 2013;28(11):3215-9.

30. Liu RH, Kang X, Xu LP, Nian HL, Yang XW, Shi HT, Wang XJ. Effects of the combined extracts of Herba Epimedii and Fructus Ligustri Lucidi on bone mineral content and bone turnover in osteoporotic rats. BMC Complement Altern Med. 2015;15:112
31. Yang YG, Tian WM, Zhang H, Li M, Shang YX. Nerve growth factor exacerbates allergic lung inflammation and airway remodeling in a rat model of chronic asthma. Exp Ther Med. 2013;6(5):1251-8.

32. Chen $M, L \vee Z$, Jiang $S$. The effects of triptolide on airway remodelling and transforming growth factor- $\beta 1 / \mathrm{Smad}$ signalling pathway in ovalbumin-sensitized mice. Immunology. 2011;132(3):376-84.

33. Komai M, Tanaka H, Masuda T, Nagao K, Ishizaki M, Sawada M, Nagai H. Role of Th2 responses in the development of allergen induced airway remodelling in a murine model of allergic asthma. $\mathrm{Br} J$ Pharmacol. 2003;138(5):912-20.

34. Dekkers BG, Maarsingh H, Meurs H, Gosens R. Airway structural components drive airway smooth muscle remodeling in asthma. Proc Am Thorac Soc. 2009:6(8):683-92.

35. Tanaka H, Masuda T, Tokuoka S, Komai M, Nagao M, Takahashi Y, Nagai H. The effect of allergen-induced airway inflammation on airway remodeling in a murine model of allergic asthma. Inflamm Res. 2001;50(12):616-24.

36. Padrid P, Snook S, Finucane T, Shiue P, Cozzi P, Solway J, Leff AR. Persistent airway hyperresponsiveness and histologic alterations after chronic antigen challenge in cats. Am J Respir Crit Care Med. 1995;151(1):184-93.

37. Sandford AJ. Asthma susceptibility: the role of transforming growth factor beta1. Respirology. 2010;15(4):583-4.

38. Bossé Y, Stankova J, Rola-Pleszczynski M. Transforming growth factor-beta1 in asthmatic airway smooth muscle enlargement: is fibroblast growth factor-2 required? Clin Exp Allergy. 2010;40(5):710-24.

39. Mirzamani MS, Nourani MR, Imani Fooladi AA, Zare S, Ebrahimi M, Yazdani S, Ghanei M, Karimfar MH. Increased expression of transforming growth factor- $\beta$ and receptors in primary human airway fibroblasts from chemical inhalation patients. Iran J Allergy Asthma Immunol. 2013;12(2):144-52.

40. Bateman ED, Hurd SS, Barnes PJ, Bousquet J, Drazen JM, FitzGerald M, Gibson P, Ohta K, O'Byrne P, Pedersen SE, Pizzichini E, Sullivan SD, Wenzel $\mathrm{SE}, \mathrm{Zar} \mathrm{HJ}$. Global strategy for asthma management and prevention: GINA executive summary. Eur Respir J. 2008;31(1):143-78.

41. Behndig AF, Larsson N, Brown JL, Stenfors N, Helleday R, Duggan ST, Dove RE, Wilson SJ, Sandstrom T, Kelly FJ, Mudway IS, Blomberg A. Proinflammatory doses of diesel exhaust in healthy subjects fail to elicit equivalent or augmented airway inflammation in subjects with asthma. Thorax. 2011;66(1):12-9.

42. Wu LC, Zarrin AA. The production and regulation of IgE by the immune system. Nat Rev Immunol. 2014;14(4):247-59.

43. Davila I, Valero A, Entrenas LM, Valveny N, Herráez L, SIGE Study Group. Relationship between serum total lgE and disease severity in patients with allergic asthma in Spain. J Investig Allergol Clin Immunol. 2015;25(2):120-7.

44. Tanaka A, Jinno M, Hirai K, Miyata Y, Mizuma H, Yamaguchi M, Ohta S, Watanabe Y, Yamamoto M, Suzuki S, Yokoe T, Adachi M, Sagara H. Longitudinal increase in total IgE levels in patients with adult asthma: an association with poor asthma control. Respir Res. 2014;15(1):144.

45. Wenzel S, Wilbraham D, Fuller R, Getz EB, Longphre M. Effect of an interleukin-4 variant on late phase asthma response to allergen challenge in asthmatic patients: results of two phase II studies. Lancet. 2007;370(9596): 1422-31.

46. Rogala B, Bozek A, Gluck J, Jarzab J. Prevalence of IgE-mediated allergy and evaluation of Th1/Th2 cytokine profiles in patients with severe bronchial asthma. Postepy Dermatol Alergol. 2015;32(4):274-80.

47. Hellman C, Lönnkvist K, Hedlin G, Halldén G, Lundahl J. Down-regulated IL-5 receptor expression on peripheral blood eosinophils from budesonide-treated children with asthma. Allergy. 2002;57(4):323-8.

48. Bergeron C, Tulic MK, Hamid Q. Airway remodelling in asthma: from benchside to clinical practice. Can Respir J. 2010;17(4):e85-93.

49. Hassan M, Jo T, Risse PA, Tolloczko B, Lemière C, Olivenstein R, Hamid Q, Martin JG. Airway smooth muscle remodeling is a dynamic process in severe long-standing asthma. J Allergy Clin Immunol. 2010;125(5):1037-45.

50. Bergeron C, Al-Ramli W, Hamid Q. Airway remodeling in asthma. Proc Am Thorac Soc. 2009;6(3):301-5.

51. Laurent GJ, McAnulty RJ, Corrin B, Cockerill P. Biochemical and histological changes in pulmonary fibrosis induced in rabbits with intratracheal bleomycin. Eur J Clin Investig. 1981;11(6):441-8.

52. Reinhardt AK, Bottoms SE, Laurent GJ, McAnulty RJ. Quantification of collagen and proteoglycan deposition in a murine model of airway remodeling. Respir Res. 2005;6(1):30.

53. Chen $M, L v Z$, Jiang $S$. The effects of triptolide on airway remodeling and transforming growth factor-1/Smad signaling pathway in ovalbumin- sensitized mice. Immunology. 2011;132(3):376-84. 
54. Bottoms SE, Howell JE, Reinhardt AK, Evans IC, McAnulty RJ. TGF- $\beta$ isoform specifi $\mathrm{c}$ regulation of airway inflammation and remodeling in a murine model of asthma. PLoS One. 2010;5(3):e9674.

55. Boxall C, Holgate ST, Davies DE. The contribution of transforming growth factor- $\beta$ and epidermal growth factor signalling to airway remodelling in chronic asthma. Eur Respir J. 2006;27(1):208-29.

56. Ma Y, Huang W, Liu C, Li Y, Xia Y, Yang X, Sun W, Bai H, Li Q, Peng Z. Immunization against TGF- $\beta 1$ reduces collagen deposition but increases sustained inflammation in a murine asthma model. Hum Vaccin Immunother. 2016;12(7):1876-85.

57. Thompson HG, Mih JD, Krasieva TB, Tromberg BJ, George SC. Epithelial-derived TGF-beta2 modulates basal and wound-healing subepithelial matrix homeostasis. Am J Physiol Lung Cell Mol Physiol. 2006;291(6):L1277-85.

58. Serpero L, Petecchia L, Sabatini F, Giuliani M, Silvestri M, Di Blasi P, Rossi GA. The effect of transforming growth factor (TGF)-beta1 and (TGF)-beta2 on nasal polyp fibroblast activities involved upper airway remodeling: modulation by fluticasone propionate. Immunol Lett. 2006;105(1):61-7.

59. Tschumperlin DJ, Shively JD, Kikuchi T, Drazen JM. Mechanical stress triggers selective release of fibrotic mediators from bronchial epithelium. Am J Respir Cell Mol Biol. 2003;28(2):142-9.

60. Kowalewski R, Malkowski A, Sobolewski K, Gacko M. Evaluation of transforming growth factor-beta signaling pathway in the wall of normal and varicose veins. Pathobiology. 2010;77(1):1-6.

61. Lv ZD, Na D, Ma XY, Zhao C, Zhao WJ, Xu HM. Human peritoneal mesothelial cell transformation into myofi broblasts in response to TGF- $\beta 1$ in vitro. Int J Mol Med. 2011:27(2):187-93.

62. Lan HY. Diverse roles of TGF- $\beta / S$ mads in renal fibrosis inflammation. Int J Biol Sci. 2011;7(7):1056-67.

63. Chakir J, Shannon J, Molet S, Fukakusa M, Elias J, Laviolette M, Boulet LP, Hamid Q. Airway remodelingassociated mediators in moderate to severe asthma: Effect of steroids on TGF- $\beta$, IL-11, IL-17, and type I and type III collagen expression. J Allergy Clin Immunol. 2003;111(6):1293-8.

64. Minshall E, Chakir J, Laviolette M, Molet S, Zhu Z, Olivenstein R, Elias JA, Hamid Q. IL-11 expression is increased in severe asthma: association with epithelial cells and eosinophils. J Allergy Clin Immunol. 2000;105(2 Pt 1):232-8.

65. Zhou J, Wu J, Chen X, Fortenbery N, Eksioglu E, Kodumudi KN, Pk EB, Dong J, Djeu JY, Wei S. Icariin and its derivative, ICT, exert anti-inflammatory, anti-tumor effects, and modulate myeloid derived suppressive cells (MDSCs) functions. Int Immunopharmacol. 2011;11(7):890-8.

66. Chen Y, Sun T, Wu J, Kalionis B, Zhang C, Yuan D, Huang J, Cai W, Fang H, Xia S. Icariin intervenes in cardiac inflammaging through upregulation of SIRT6 enzyme activity and inhibition of the NF-kappa B pathway. Biomed Res Int. 2015;2015:895976.

67. Hu B, Du Q, Deng S, An HM, Pan CF, Shen KP, Xu L, Wei MM, Wang SS. Ligustrum Lucidum Ait. Fruit extract induces apoptosis and cell senescence in human hepatocellular carcinoma cells through upregulation of p21. Oncol Rep. 2014;32(3):1037-42.

68. Lin HM, Yen FL, Ng LT, Lin CC. Protective effects of Ligustrum Lucidum fruit extract on acute butylated hydroxytoluene-induced oxidative stress in rats. J Ethnopharmacol. 2007:111(1):129-36.

69. Wang J, Shan A, Liu T, Zhang C, Zhang Z. In vitro immunomodulatory effects of an oleanolic acid-enriched extract of Ligustrum Lucidum fruit (Ligustrum Lucidum supercritical $\mathrm{CO}_{2}$ extract) on piglet immunocytes. Int Immunopharmacol. 2012;14(4):758-63

\section{Submit your next manuscript to BioMed Central and we will help you at every step:}

- We accept pre-submission inquiries

- Our selector tool helps you to find the most relevant journal

- We provide round the clock customer support

- Convenient online submission

- Thorough peer review

- Inclusion in PubMed and all major indexing services

- Maximum visibility for your research

Submit your manuscript at www.biomedcentral.com/submit

CBiomed Central 